Research Paper

\title{
Left Atrial Diameter and Albumin with Renal Outcomes in Chronic Kidney Disease
}

\author{
Szu-Chia Chen ${ }^{1,3,5}$, Jer-Ming Chang 1,3,4, Yi-Chun Tsai ${ }^{1,3}$, Jiun-Chi Huang1,3, Ho-Ming Su ${ }^{2,3,5 凶}$, Shang-Jyh \\ Hwang ${ }^{1,4}$, Hung-Chun Chen ${ }^{1,4}$ \\ 1. Division of Nephrology, Department of Internal Medicine, Kaohsiung Medical University Hospital, Kaohsiung Medical University, \\ Kaohsiung, Taiwan; \\ 2. Division of Cardiology, Department of Internal Medicine, Kaohsiung Medical University Hospital, Kaohsiung Medical University, \\ Kaohsiung, Taiwan; \\ 3. Department of Internal Medicine, Kaohsiung Municipal Hsiao-Kang Hospital, Kaohsiung Medical University; \\ 4. Faculty of Renal Care, College of Medicine, Kaohsiung Medical University, Kaohsiung, Taiwan \\ 5. Faculty of Medicine, College of Medicine, Kaohsiung Medical University, Kaohsiung, Taiwan
}

$\triangle$ Corresponding author: Ho-Ming Su, M.D. Department of Internal Medicine, Kaohsiung Municipal Hsiao-Kang Hospital, Kaohsiung Medical University. 482, Shan-Ming Rd., Hsiao-Kang Dist., 812 Kaohsiung, Taiwan, R.O.C. TEL: 886- 7- 8036783 - 3441, FAX: 886- 7- 8063346 E-mail: cobeshm@seed.net.tw.

(c) Ivyspring International Publisher. This is an open-access article distributed under the terms of the Creative Commons License (http://creativecommons.org/ licenses/by-nc-nd/3.0/). Reproduction is permitted for personal, noncommercial use, provided that the article is in whole, unmodified, and properly cited.

Received: 2013.01.09; Accepted: 2013.03.13; Published: 2013.03.16

\begin{abstract}
Background and Aim: Echocardiographic left atrial diameter (LAD) has been documented to be an independent predictor of adverse cardiovascular outcomes in various populations. An enlarged left atrium is frequently noted in chronic kidney disease (CKD). We examined the association between albumin and indexed LAD (indexed to height) and assessed whether the combination of indexed LAD and albumin was independently associated with renal outcomes in patients with CKD stages 3-5.

Methods: This longitudinal study enrolled 395 patients, who were classified into four groups according to median values of indexed LAD (LAD/height) and albumin. The change in renal function was measured by estimated glomerular filtration rate (eGFR) slope. Rapid renal progression was defined as eGFR slope less than $-3 \mathrm{ml} / \mathrm{min} / 1.73 \mathrm{~m}^{2} / y e a r$. The renal end point was defined as commencement of dialysis.

Results: Albumin was significantly associated with indexed LAD $(\beta=-0.108, P=0.024)$. During follow-up period, seventy-four patients started dialysis. After the multivariate analysis, the group with higher indexed LAD and lower albumin was independently associated with rapid renal progression (odds ratio, 7.979; 95\% confidence interval [Cl], 3.028 to 21.025 ) and progression to dialysis (hazard ratio, $2.352 ; 95 \% \mathrm{Cl}, 1.078$ to 5.131 ).

Conclusions: Our findings show that albumin is independently associated with indexed LAD and suggest that the combination of increased indexed LAD and hypoalbuminemia is independently associated with rapid renal progression and progression to dialysis in patients with CKD. Assessments of serum albumin and indexed LAD by echocardiography are useful for predicting the risk for adverse renal outcomes.
\end{abstract}

Key words: indexed left atrial diameter, albumin, chronic kidney disease, renal function progression.

\section{Introduction}

M-mode left atrial (LA) dimension is simple, convenient, and commonly used in clinical practice and research studies (1). LA enlargement detected by echocardiography is frequently noted in patients with 
chronic kidney disease (CKD) due to pressure and volume overload (2). Recently, an enlarged left atrium has been considered a marker of adverse cardiovascular outcomes in chronic renal failure patients $(3,4)$. However, published data of LA size on renal outcomes in CKD is limited and inconsistent. One study found that LA volume index was an independent risk factor for the period of time before dialysis in patients with CKD stages 4-5 (5), whereas another study suggested only a weak association between LA size and renal outcome in CKD subjects (6). Thus, the status of LA enlargement as an independent risk marker for renal progression remains controversial.

Previous studies had identified several risk factors for LA enlargement, including overweight, increased systolic and pulse pressures, duration of hypertension, and inflammatory markers (7-9). In report by Kursat et al, they found the malnutrition index, calculated using Subjective Global Assessment, had a positive correlation with LA diameter (LAD) (10). They explained their findings by inadequate volume control. The result suggested that serum albumin, a well-known nutrition marker, might be related to LAD. Low serum albumin level had also been reported to be a significant predictor for progression of renal function through the mechanism of inflammation. Inflammation contributed to the progression of CKD by inducing the release of cytokines and the increased production and activity of adhesion molecules, which together contribute to $\mathrm{T}$ cell adhesion and migration into the interstitium, subsequently attracting pro-fibrotic factors $(11,12)$.

The hypothesis that the combination of LAD and serum albumin is a strong predictor of adverse renal outcomes in CKD patients has never been examined. In this study, we investigate the relationship between indexed LAD (indexed to height) (13) and serum albumin in patients with CKD stages 3-5. Furthermore, we also assessed whether the combination of indexed LAD and serum albumin was independently associated with rapid renal progression and progression to dialysis in CKD subjects.

\section{Subjects and Methods}

\section{Study Patients and Design}

The study was conducted in a regional hospital in southern Taiwan. We consecutively enrolled 518 pre-dialysis patients with stages 3 to 5 of CKD according to the National Kidney Foundation-Kidney Disease Outcomes Quality Initiative (K/DOQI) guidelines (14) from our Outpatient Department of Internal Medicine from January 2007 to May 2010. We classified our patients with evidence of kidney damage lasting for more than 3 months into CKD stages 3 ,
4 , and 5, based on estimated glomerular filtration rate (eGFR) level (mL/min/1.73 $\left.\mathrm{m}^{2}\right)$ of 30 to 59,15 to 29 , and $<15$, respectively. Three patients with significant mitral valve disease and 5 patients with inadequate image visualization were excluded. Five patients refused echocardiography examinations due to personal reasons. Fifty-one patients with less than three eGFR measurements during the follow-up period were excluded. In addition, those patients with mortality $(\mathrm{n}=$ 9) or entering dialysis therapy $(n=30)$ within 3 months after enrollment were also excluded to avoid incomplete observation of change in renal function. Because atrial fibrillation would influence LAD, patients with atrial fibrillation $(n=20)$ were also excluded. Finally, 395 patients (mean age $66.6 \pm 12.1$ years, 265 males) were included in this study. The protocol was approved by our Institutional Review Board and all enrolled patients gave written, informed consent.

\section{Evaluation of cardiac structure and function}

The echocardiographic examination was performed by two experienced cardiologists with a VIVID 7 (General Electric Medical Systems, Horten, Norway), with the participant respiring quietly in the left decubitus position. The cardiologists were blind to the other data. Two-dimensional and two-dimensionally guided M-mode images were recorded from the standardized views. The echocardiographic measurements included LAD, left ventricular internal diameter in diastole (LVIDd), left ventricular posterior wall thickness in diastole (LVPWTd), interventricular septal wall thickness in diastole (IVSTd), peak early transmitral filling wave velocity (E) and peak late transmitral filling wave velocity (A), E-wave deceleration time (EDT) and early diastolic mitral velocity (Ea). LAD was indexed to height (LAD/height). Left ventricular systolic function was assessed by left ventricular ejection fraction (LVEF). Left ventricular mass was calculated using Devereux-modified method, i.e. left ventricular mass $=1.04 \times[($ IVSTd + LVIDd + LVPWTd $)^{3}-$ LVIDd $^{3}$ ] - 13.6g (15). Left ventricular mass index (LVMI) was calculated by dividing left ventricular mass by body surface area. Left ventricular hypertrophy $(\mathrm{LVH})$ was defined as suggested by the 2007 European Society of Hypertension/European Society of Cardiology guidelines (16).

\section{Collection of demographic, medical, and la- boratory data}

Demographic and medical data including age, gender, smoking history (ever versus never), and comorbid conditions were obtained from medical records or interviews with patients. The body mass index (BMI) was calculated as the ratio of weight in 
kilograms divided by square of height in meters. Laboratory data were measured from fasting blood samples using an autoanalyzer (Roche Diagnostics $\mathrm{GmbH}$, D-68298 Mannheim COBAS Integra 400). Serum creatinine was measured by the compensated Jaffé (kinetic alkaline picrate) method in a Roche/Integra 400 Analyzer (Roche Diagnostics, Mannheim, Germany) using a calibrator traceable to isotope-dilution mass spectrometry (17). The value of eGFR was calculated using the 4-variable equation in the Modification of Diet in Renal Disease (MDRD) study (18). Proteinuria was examined by dipsticks (Hema-Combistix, Bayer Diagnostics). A test result of $1+$ or more was defined as positive. Blood and urine samples were obtained within 1 month of enrollment. In addition, information regarding patient medications including aspirin, angiotensin converting enzyme inhibitors (ACEIs), angiotensin II receptor blockers (ARBs), non ACEI/ARB antihypertensive drugs, and HMG-CoA reductase inhibitors (statins) during the study period was obtained from medical records.

\section{Assessment of rate of renal function decline and definition of rapid renal progression}

The rate in renal function decline was assessed by the eGFR slope, defined as the regression coefficient between eGFR and time in unit of $\mathrm{ml} / \mathrm{min} / 1.73$ $\mathrm{m}^{2} /$ year. At least three eGFR measurements after echocardiographic examination were required to estimate eGFR slope. Any reduction greater than 3 $\mathrm{ml} / \mathrm{min} / 1.73 \mathrm{~m}^{2} /$ year, i.e. slope more negative than -3 $\mathrm{ml} / \mathrm{min} / 1.73 \mathrm{~m}^{2} /$ year $(<-3)$, was considered as rapid renal progression (19).

\section{Definition of renal end point}

The renal end point was defined as commencement of dialysis. In patients reaching renal end point, renal function data were censored at the start of renal replacement therapy. The other patients were followed until February 2011. The commencement of dialysis was determined according to the regulations by the National Health Insurance for dialysis therapy based on laboratory data, nutrition status, and uremic symptoms and signs.

\section{Reproducibility}

Thirty patients were randomly selected for evaluation of the interobserver variability of LAD measurement by 2 independent observers. To get the intraobsever variability, the same measurements were repeated 1 week apart. Mean percent error was calcu- lated as the absolute difference divided by the average of the two observations.

\section{Statistical analysis}

Statistical analysis was performed using SPSS version 12.0 (SPSS Inc., Chicago, IL, USA) for windows. Data are expressed as percentages, mean \pm standard deviation, mean \pm standard error of mean for eGFR slope, or median $\left(25^{\text {th }}-75^{\text {th }}\right.$ percentile) for triglyceride and number of serum creatinine measurements.

The study patients were stratified into 4 groups according to median values of indexed LAD and albumin. Multiple comparisons among the study groups were performed by one-way analysis of variance (ANOVA) followed by post hoc test adjusted with a Bonferroni correction. The relationship between two continuous variables was assessed by a bivariate correlation method (Pearson's correlation). Multiple linear regression analysis was used to identify the factors associated with indexed LAD. Multiple logistic regression analysis was employed to identify the risk factors associated with rapid renal progression. Time to commencement of dialysis and covariates of risk factors were modeled using the Cox proportional hazards model. Survival curve for dialysis was derived using Cox-regression analysis. Significant variables in univariate analysis were selected for multivariate analysis. A difference was considered significant if the $P$ value was less than 0.05 .

\section{Results}

A total of three hundred and ninety-five non-dialyzed CKD patients were included. The mean age was $66.2 \pm 12.1$ years and there were 250 males and 145 females. The value of eGFR slope of all patients was $-1.59 \pm 0.14 \mathrm{ml} / \mathrm{min} / 1.73 \mathrm{~m}^{2} /$ year. The average number of serum creatinine measurements during the follow-up period was $8\left(25^{\text {th }}-75^{\text {th }}\right.$ percentile: 6-12) times. The study patients were stratified into 4 groups according to median values of indexed LAD $(2.31 \mathrm{~cm} / \mathrm{m})$ and albumin $(4.1 \mathrm{~g} / \mathrm{dL})$. The comparison of clinical characteristics among study groups was shown in Table 1. There were 113, 84, 94, and 104 patients in 4 groups, respectively. The eGFR slopes in 4 groups were $-0.54 \pm 0.23,-1.64 \pm 0.27,-1.41 \pm 0.24$, and $-2.84 \pm 0.33 \mathrm{~mL} / \mathrm{min} / 1.73 \mathrm{~m}^{2} /$ year, respectively. The eGFR slope was lower in the group with higher indexed LAD and lower albumin than in the other groups $(P \leq 0.001)$. Figure 1 illustrated the eGFR slopes among 4 study groups. 
Table I. Clinical characteristics of patients among study groups.

\begin{tabular}{|c|c|c|c|c|}
\hline Characteristics & $\begin{array}{l}\text { Lower iLAD and } \\
\text { higher albumin } \\
(\mathrm{n}=113)\end{array}$ & $\begin{array}{l}\text { Lower iLAD and } \\
\text { albumin } \\
(\mathrm{n}=84)\end{array}$ & $\begin{array}{l}\text { Higher iLAD and } \\
\text { albumin } \\
(\mathrm{n}=94)\end{array}$ & $\begin{array}{l}\text { Higher iLAD and } \\
\text { lower albumin } \\
(\mathrm{n}=104)\end{array}$ \\
\hline eGFR slope (ml/min/1.73 m²/year) & $-0.54 \pm 0.23$ & $-1.64 \pm 0.27^{*}$ & $-1.41 \pm 0.24$ & $-2.84 \pm 0.33^{*}+\#$ \\
\hline Age (year) & $63.8 \pm 12.5$ & $70.0 \pm 11.5^{*}$ & $64.3 \pm 11.2^{\dagger}$ & $67.6 \pm 12.3$ \\
\hline Male gender $(\%)$ & 69.9 & 64.3 & 55.3 & 62.5 \\
\hline Smoking history $(\%)$ & 31.9 & 36.9 & 22.3 & 31.7 \\
\hline Diabetes mellitus (\%) & 43.4 & 53.6 & $62.8^{*}$ & $67.3^{*}$ \\
\hline Hypertension (\%) & 77.9 & 73.8 & $90.4^{\dagger}$ & $87.5^{\dagger}$ \\
\hline Coronary artery disease (\%) & 8.0 & 7.1 & 12.8 & 15.4 \\
\hline Cerebrovascular disease (\%) & 11.5 & 11.9 & 12.8 & 22.1 \\
\hline \multicolumn{5}{|l|}{ Stage of CKD } \\
\hline Stage $3(\%)$ & 55.8 & 44.0 & 41.5 & $26.0^{*}+$ \\
\hline Stage $4(\%)$ & 28.3 & 34.5 & 29.8 & 31.7 \\
\hline Stage $5(\%)$ & 15.9 & 21.4 & 28.7 & 42.3 \\
\hline Mean arterial pressure $(\mathrm{mmHg})$ & $98.9 \pm 11.1$ & $97.0 \pm 13.2$ & $100.0 \pm 12.9$ & $101.4 \pm 14.5$ \\
\hline Pulse pressure $(\mathrm{mmHg})$ & $56.5 \pm 12.9$ & $61.8 \pm 19.4$ & $61.6 \pm 17.4$ & $67.6 \pm 17.9^{*}$ \\
\hline Body mass index $\left(\mathrm{kg} / \mathrm{m}^{2}\right)$ & $24.2 \pm 3.1$ & $23.7 \pm 3.8$ & $27.2 \pm 3.6^{*} \dagger$ & $26.5 \pm 4.1^{* \dagger}$ \\
\hline \multicolumn{5}{|l|}{ Laboratory parameters } \\
\hline $\operatorname{Albumin}(\mathrm{g} / \mathrm{dL})$ & $4.29 \pm 0.16$ & $3.85 \pm 0.26^{*}$ & $4.32 \pm 0.18^{\dagger}$ & $3.76 \pm 0.30^{\star \star \#}$ \\
\hline Fasting glucose $(\mathrm{mg} / \mathrm{dL})$ & $115.1 \pm 47.8$ & $132.7 \pm 59.3$ & $131.1 \pm 52.0$ & $132.2 \pm 75.2$ \\
\hline Triglyceride (mg/dL) & $132(91.193)$ & $125.5(93-182)$ & $152(108-236)$ & $149(98-226)$ \\
\hline Total cholesterol (mg/dL) & $188.1 \pm 43.4$ & $196.1 \pm 46.7$ & $195.4 \pm 48.3$ & $201.4 \pm 47.0$ \\
\hline Hemoglobin $(\mathrm{g} / \mathrm{dL})$ & $12.5 \pm 2.2$ & $11.2 \pm 1.8^{*}$ & $12.0 \pm 2.2$ & $11.0 \pm 2.4^{* \#}$ \\
\hline Baseline eGFR $\left(\mathrm{mL} / \mathrm{min} / 1.73 \mathrm{~m}^{2}\right)$ & $31.4 \pm 13.3$ & $27.5 \pm 12.9$ & $27.0 \pm 14.8$ & $22.0 \pm 13.0^{* \#}$ \\
\hline Calcium-phosphorous product $\left(\mathrm{mg}^{2} / \mathrm{dL}^{2}\right)$ & $36.5 \pm 7.6$ & $37.7 \pm 9.1$ & $39.0 \pm 7.8$ & $39.5 \pm 8.8$ \\
\hline Uric acid (mg/dL) & $8.0 \pm 1.9$ & $7.9 \pm 2.0$ & $8.1 \pm 2.0$ & $8.2 \pm 2.2$ \\
\hline Proteinuria (\%) & 51.8 & 65.1 & 67.0 & $75.0^{*}$ \\
\hline \multicolumn{5}{|l|}{ Medications } \\
\hline Aspirin use (\%) & 20.9 & 21.7 & 33.0 & 26.7 \\
\hline ACEI and/or ARB use (\%) & 76.4 & 72.3 & 77.7 & 70.3 \\
\hline Non-ACEI/ARB antihypertensive drug use (\%) & 61.1 & 59.5 & $87.2^{*}+$ & $78.8^{*}+$ \\
\hline Statin use $(\%)$ & 21.8 & 30.1 & 20.2 & 30.7 \\
\hline \multicolumn{5}{|l|}{ Echocardiographic data } \\
\hline LAD/height $(\mathrm{cm} / \mathrm{m})$ & $2.00 \pm 0.23$ & $1.99 \pm 0.22$ & $2.64 \pm 0.24^{*} \dagger$ & $2.64 \pm 0.24^{*} \dagger$ \\
\hline $\operatorname{LVMI}\left(\mathrm{g} / \mathrm{m}^{2}\right)$ & $118.4 \pm 37.0$ & $126.3 \pm 41.9$ & $150.1 \pm 48.7^{*+}$ & $159.0 \pm 50.7^{*}+$ \\
\hline LVH (\%) & 41.6 & 50.0 & $77.7^{*}+$ & $79.8^{*}$ \\
\hline LVEF (\%) & $71.0 \pm 9.5$ & $68.6 \pm 9.2$ & $69.2 \pm 9.4$ & $66.9 \pm 12.4^{*}$ \\
\hline $\mathrm{E} / \mathrm{A}$ & $0.86 \pm 0.26$ & $0.74 \pm 0.28$ & $0.83 \pm 0.30$ & $0.92 \pm 0.40^{\dagger}$ \\
\hline EDT (ms) & $215.8 \pm 54.6$ & $249.1 \pm 69.7^{*}$ & $225.2 \pm 62.4$ & $220.8 \pm 61.5^{\dagger}$ \\
\hline $\mathrm{E} / \mathrm{Ea}$ & $7.8 \pm 2.4$ & $9.9 \pm 4.1^{*}$ & $10.1 \pm 4.5^{*}$ & $12.8 \pm 5.8^{* \star \#}$ \\
\hline
\end{tabular}

Abbreviations. iLAD, indexed left atrial diameter; LAD, left atrial diameter; eGFR, estimated glomerular filtration rate; CKD, chronic kidney disease; ACEI, angiotensin converting enzyme inhibitor; ARB, angiotensin II receptor blocker; LVMI, left ventricular mass index; LVH, left ventricular hypertrophy; LVEF, left ventricular ejection fraction; E, peak early transmitral filling wave velocity; A, peak late transmitral filling wave velocity; EDT, E-wave deceleration time; Ea, early diastolic velocity of lateral mitral annulus. ${ }^{*} P<0.05$ compared with lower LAD and higher albumin; $+P<0.05$ compared with lower LAD and albumin; ${ }^{\#} P<$ 0.05 compared with higher LAD and albumin. 


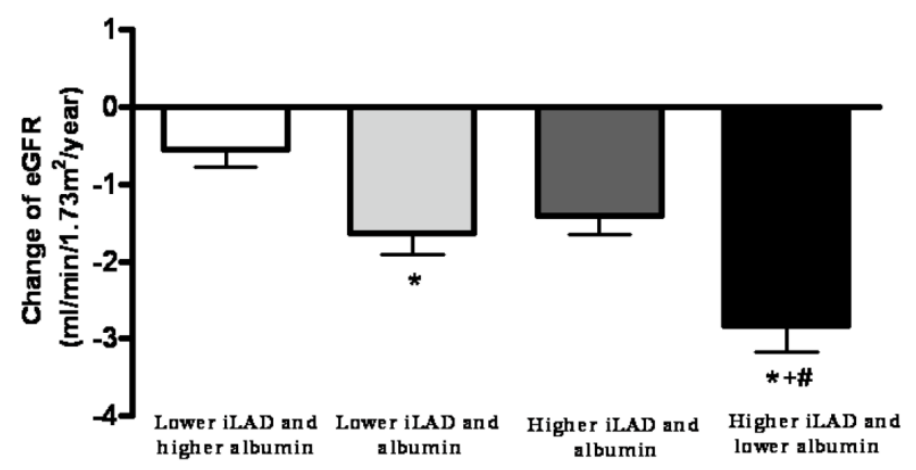

Figure I. The estimated glomerular filtration rate (eGFR) slopes among 4 study groups. The eGFR slope was lower in the group with higher indexed left atrial diameter (iLAD) and lower albumin than in the other groups. ${ }^{*} P<0.05$ compared with the group with lower iLAD and higher albumin; $\uparrow P<0.05$ compared with the group with lower iLAD and albumin; $\# P<0.05$ compare with the group with higher iLAD and albumin.

\section{Determinants of indexed LAD}

Table 2 shows the determinants of indexed LAD in our study patients. The indexed LAD correlated positively with diabetes mellitus (DM), hypertension, coronary artery disease, mean arterial pressure, pulse pressure, BMI, fasting glucose, triglyceride, calcium-phosphorous product, proteinuria and non-ACEI/ARB antihypertensive drug use but negatively with male, albumin, hemoglobin and baseline eGFR. Further forward multivariate analysis showed that increased indexed LAD was correlated with high BMI $(\beta=0.354, P<0.001)$, high pulse pressure $(\beta=$ $0.104, P=0.034)$, low albumin $(\beta=-0.108, P=0.024)$, low hemoglobin $(\beta=-0.128, \quad P=0.011)$, and non-ACEI/ARB antihypertensive drug use $(\beta=0.231$, $P<0.001)$. In addition, indexed LAD was correlated with LVMI $(r=0.430, P<0.001), \mathrm{LVH}(r=0.371, P<$ $0.001), \mathrm{E} / \mathrm{A}$ ratio $(r=-0.155, P=0.002)$, and $\mathrm{E} / \mathrm{Ea}$ ratio $(r=0.388, P<0.001)$.

Table 2. Determinants of indexed left atrial diameter (indexed to height) using multivariate forward linear analysis in study patients.

\begin{tabular}{lll}
\hline Parameter & \multicolumn{2}{l}{ Multivariate (Forward) } \\
\cline { 2 - 3 } & $\begin{array}{l}\text { Standardized } \\
\text { coefficient } \beta\end{array}$ & $\mathrm{P}$ \\
\hline Pulse pressure $(\mathrm{mmHg})$ & 0.104 & 0.034 \\
Body mass index $(\mathrm{kg} / \mathrm{m} 2)$ & 0.354 & $<0.001$ \\
Albumin $(\mathrm{g} / \mathrm{dL})$ & -0.108 & 0.024 \\
Hemoglobin $(\mathrm{g} / \mathrm{dL})$ & -0.128 & 0.011 \\
$\begin{array}{l}\text { Non-ACEI/ARB antihypertensive } \\
\text { drug use }\end{array}$ & 0.231 & $<0.001$ \\
\end{tabular}

Values expressed as standardized coefficient $\beta$. Abbreviations are the same as in Table 1. Adjusted for gender, diabetes mellitus, hypertension, coronary artery disease, mean arterial pressure, pulse pressure, body mass index, albumin, fasting glucose, triglyceride, hemoglobin, baseline eGFR, calcium-phosphorous product, proteinuria and non-ACEI/ARB antihypertensive drug use

\section{Risk of rapid renal progression}

Table 3 showed the determinants of rapid renal progression (eGFR slope $<-3 \mathrm{ml} / \mathrm{min} / 1.73 \mathrm{~m}^{2} /$ year) among study patients. In the univariate regression analysis, rapid renal progression was found to be significantly associated with DM, coronary artery disease, cerebrovascular disease, high mean arterial pressure, high pulse pressure, the groups with lower indexed LAD and albumin and higher indexed LAD and lower albumin (versus lower indexed LAD and higher albumin), high fasting glucose, high total cholesterol, low hemoglobin, low baseline eGFR, high calcium-phosphorous product, high uric acid, proteinuria, non-ACEI/ARB antihypertensive drug use, low LVEF, high LVMI and high E/Ea ratio. In the multiple forward logistic regression analysis, the group with higher indexed LAD and lower albumin (odds ratio [OR], 7.979; 95\% confidence interval [CI], 3.028 to $21.025 ; P<0.001$ ) and proteinuria were independent risk factors for rapid renal progression.

\section{Risk of progression to dialysis}

The mean follow-up period was $27.2 \pm 11.1$ months (range 3.3 to 50.0 months). During the period of follow-up, seventy-four patients $(18.7 \%)$ started hemodialysis. Table 4 shows a Cox proportional hazards regression analysis for progression to commencement of dialysis. The univariate regression analysis shows that female, hypertension, cerebrovascular disease, high pulse pressure, the group with higher indexed LAD and lower albumin (versus lower indexed LAD and higher albumin), low hemoglobin, low baseline eGFR, high calcium-phosphorous product, proteinuria, ACEI and/or ARB use, non-ACEI/ARB antihypertensive drug use, high LVMI and high E/Ea ratio were associated with a significant increase in progression to commencement of dialysis. In the multivariate forward analysis, the 
group with higher indexed LAD and lower albumin (hazard ratio [HR], 2.352; 95\% CI, 1.078 to 5.131; $P=$ 0.032) was positively associated with and baseline eGFR was negatively associated with progression to commencement of dialysis. Figure 2 illustrated the adjusted Cox regression survival curves for dialysis-free survival among 4 study groups. The group with higher indexed LAD and lower albumin had a worse dialysis-free survival than that with lower indexed LAD and higher albumin.

The intraobserver and interobserver mean percent errors for LAD measurement were $2.2 \pm 2.0 \%$ and $2.7 \pm 2.1 \%$, respectively.

Because the indexed LAD was correlated positively with BMI, we further performed analysis using LAD as a variable. We found the similar finding that the group with higher LAD and lower albumin (versus lower LAD and higher albumin) was independently associated with rapid renal progression (OR, 9.824; 95\% CI, 4.022 to 23.995; $P<0.001$ ), and progression to commencement of dialysis (HR, 2.337; 95\% CI, 1.154 to 4.732; $P=0.018$ ) after the multivariate forward analysis.

We further analyzed the association between the study groups and cardiovascular events. Cardiovascular events were defined as cardiovascular death, hospitalization for unstable angina, nonfatal myocardial infarction, sustained ventricular arrhythmia, hospitalization for congestive heart failure, transient ischemia attack, and stroke. The univariate regression analysis showed that the group with higher indexed LAD and lower albumin (HR 3.043, 95\% CI 1.477 to $6.270, P=0.003$ versus lower indexed LAD and higher albumin) was associated with an increase in cardiovascular events.

Table 3. Determinants of rapid renal progression (eGFR lope $<-3 \mathrm{ml} / \mathrm{min} / 1.73 \mathrm{~m}^{2} /$ year) using multivariate forward logistic analysis in study patients.

\begin{tabular}{|c|c|c|}
\hline \multirow[t]{2}{*}{ Parameter } & \multicolumn{2}{|c|}{ Multivariate (Forward) } \\
\hline & OR $(95 \% \mathrm{CI})$ & $P$ \\
\hline \multicolumn{3}{|l|}{ Study groups } \\
\hline Lower iLAD and higher albumin & Reference & \\
\hline Lower iLAD and albumin & $2.833(0.962-8.342)$ & 0.059 \\
\hline Higher iLAD and albumin & $1.390(0.456-4.237)$ & 0.563 \\
\hline Higher iLAD and lower albumin & $7.979(3.028-21.025)$ & $<0.001$ \\
\hline Proteinuria & 4.646 (1.947-11.068) & 0.001 \\
\hline
\end{tabular}

Table 4. Predictors of progression to dialysis using Cox proportional hazards model.

\begin{tabular}{lll}
\hline Parameter & Multivariate (Forward) & $P$ \\
\cline { 3 - 3 } & HR (95\% CI) & \\
\hline Study groups & Reference & 0.983 \\
Lower iLAD and higher albumin & $0.989(0.357-2.742)$ & 0.316 \\
Lower iLAD and albumin & $0.630(0.255-1.555)$ & 0.032 \\
Higher iLAD and albumin & $2.352(1.078-5.131)$ & $<0.001$ \\
Higher iLAD and lower albumin & $0.807(0.764-0.852)$ & \\
Baseline eGFR (per $\left.1 \mathrm{~mL} / \mathrm{min} / 1.73 \mathrm{~m}^{2}\right)$ & & \\
\hline
\end{tabular}

Values express as hazard ratios (HR) and 95\% confidence interval (CI). Abbreviations are the same as in Table 1. Adjusted for female, hypertension, cerebrovascular disease, pulse pressure, the group with higher iLAD and lower albumin (versus lower iLAD and higher albumin), hemoglobin, baseline eGFR, calcium-phosphorous product, proteinuria, ACEI and/or ARB use, non-ACEI/ARB antihypertensive drug use, LVMI and E/Ea. 


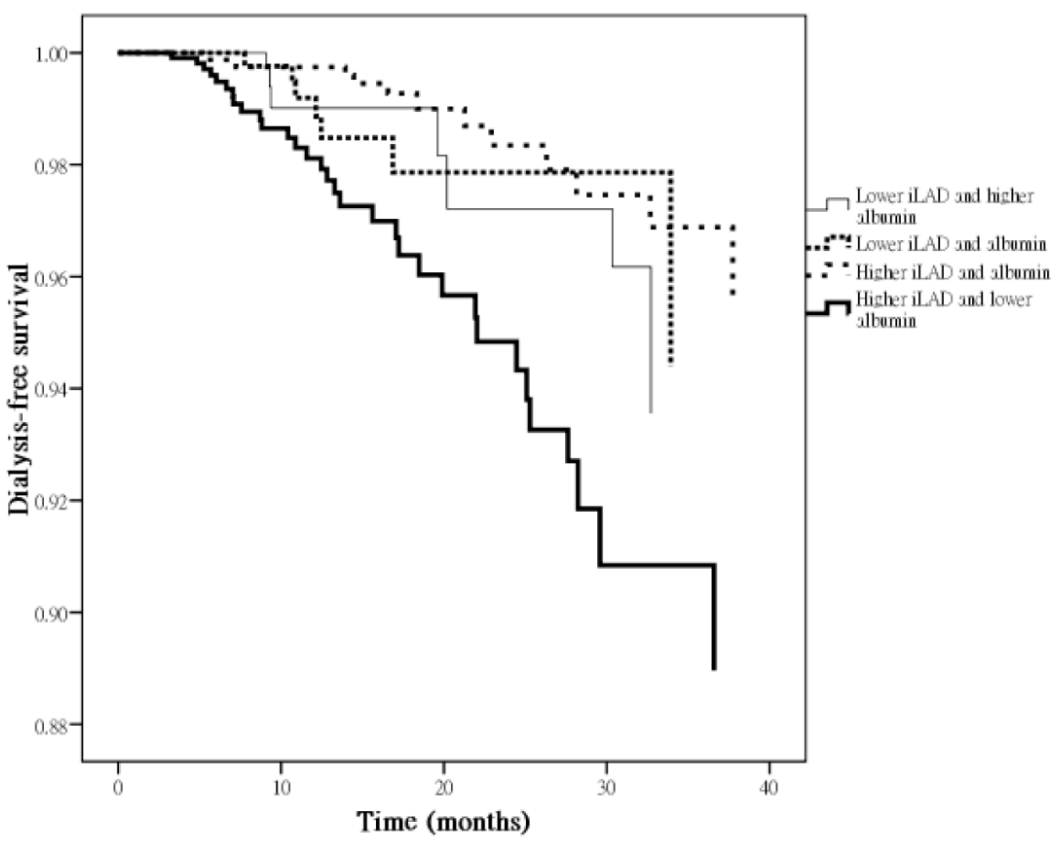

Figure 2. Adjusted dialysis-free survival curves among 4 study groups. The group with higher indexed left atrial diameter (iLAD) and lower albumin had a worse dialysis-free survival than that with lower iLAD and higher albumin.

\section{Discussion}

In the present study, we evaluated the association of the combination of indexed LAD and serum albumin on renal outcomes in patients with CKD stages $3-5$. We found that serum albumin was independently associated with indexed LAD. In addition, compared with the group with lower indexed LAD and higher albumin, the group with higher indexed LAD and lower albumin was independently associated with rapid renal progression and progression to dialysis.

Chronic fluid overload is frequently encountered in CKD patients and is associated with LVH and left ventricular dysfunction (20). Fluid overload may be involved in the pathogenesis of malnutrition (21). Previous reports had observed an independent relationship between malnutrition and extracellular fluid volume overload in dialysis patients $(10,22)$. The mechanisms to explain the correlation between fluid status and nutritional status remains inconclusive. First, fluid overload may lead to gastrointestinal edema and poor ingestion (21). Second, fluid status has an effect on inflammatory status and then inflammatory conditions can cause loss of muscle mass through activation of the ubiquitin-proteasome proteolytic system $(23,24)$. Moreover, improved fluid status is associated with improvement in nutritional status, whereas deterioration in fluid status is associ- ated with the development of malnutrition (23). A previous report had investigated the relationship between the degree of malnutrition and echocardiographic parameters in 72 hemodialysis patients (10). They found that the malnutrition index, calculated using Subjective Global Assessment, had a positive correlation with LAD. Increasing degrees of malnutrition are associated with more profound derangements in the volume status. In addition, Wang et al. (21) had evaluated the association between inadequate dietary protein intake and malnutrition in dialysis patients with a history of volume overload, which suggested a possible correlation between volume overload and malnutrition. Low serum albumin level has been regarded as malnutrition status. Volume excess may be a mechanism explaining the association between malnutrition and increased LAD. Our study consistently revealed that serum albumin was independently associated with indexed LAD. However, although our study found the correlation between hypoalbuminemia and LA enlargement, the causality could not be identified in our study.

The association between albumin and LAD may be resulted from an association of malnutrition with other risk factors such as inflammation and atherosclerosis. Malnutrition may worsen patient outcome by aggravating existing inflammation and accelerating atherosclerosis (24). Inflammation may contribute to LA enlargement through the process of endothelial 
dysfunction $(7,9,26)$. In addition, atherosclerosis directly causes a decrease in blood perfusion in the lower extremities and an increase in arterial wall stiffness, contributing to decreasing arterial distensibility and then final to LA enlargement $(27,28)$. Demirci et al. (22) had also evaluated the association between malnutrition-inflammation-atherosclerosis and volume status in peritoneal dialysis patients. They used the multi-frequency bioelectrical impedance analysis technique, a useful tool to measure body fluid, to analysis the relationship between extracellular volume and echocardiographic parameters. They found fluid overload defined by multi-frequency bioelectrical impedance analysis technique was significantly associated with low albumin, high C-reactive protein, and elevated carotid artery intima-media thickness. Besides, extracellular volume status was significantly associated with LAD and LVMI (22). In our study, we used indexed LAD as a rough surrogate of fluid status and found indexed LAD had a significant correlation with left ventricular filling pressure indicated by E/Ea. Although we also found increased indexed LAD significantly correlated with low albumin and the combination of indexed LAD and albumin was useful in identification of patients with adverse renal outcomes, further researches are still warranted to determine the association between fluid and nutrition statuses and evaluate whether the combination of fluid and nutrition statuses is useful in prediction of poor renal outcomes.

An enlarged left atrium was independently associated with adverse cardiovascular outcomes such as stroke, congestive heart failure, atrial fibrillation, and cardiovascular death in various pathologic conditions $(1,3,4,29)$. Left atrium acts as a volume sensor of the heart and its dilatation reflects a sustained elevation in left ventricular filling pressure $(30,31)$. The LA size was directly proportional to the left ventricular filling pressure (31). Besides, LA enlargement is closely related to LVH (32). Our study also revealed that increased indexed LAD was correlated with high LVMI, LVH, and high E/Ea ratio, which was consistent with previous findings.

The mechanisms of progressive renal function decline in patients with cardiac abnormalities are multi-factorial including chronic renal hypoperfusion, malnutrition, subclinical inflammation, endothelial dysfunction, accelerated atherosclerosis, increased renal vascular resistance, systemic neurohormonal factors, pharmacotherapies, and anemia $(33,34)$. Furukawa et al. (5) evaluated the factor of LA volume index in progression to hemodialysis in 140 patients with CKD stages 4-5. They found LA volume index was an independent risk factor for the period of time before dialysis. In our study, increased indexed LAD was also independently associated with rapid renal progression and progression to dialysis after adjustment of baseline eGFR. This implied that patients with an increased indexed LAD might have a high volume status and left ventricular filling pressure, thereby increasing renal efferent pressure and decreasing renal blood flow and finally causing a progressive renal function decline (33).

In our study, compared with the group with lower indexed LAD and higher albumin, the group with higher indexed LAD and lower albumin showed a condition of increased risk for renal morbidity, such as higher prevalence of DM, advanced CKD stages, higher pulse pressure, lower hemoglobin and more proteinuria. Even after adjustment for these confounding factors, the group with higher indexed LAD and lower albumin was still associated with rapid renal progression and progression to dialysis. Hence, LA enlargement and hypoalbuminemia might have a synergic effect on the risk of adverse renal outcomes.

CKD is an increasing worldwide public health problem and associated with increased morbidity and mortality. Progression to end-stage renal disease and development of cardiovascular disease are two major poor outcomes of CKD (35). We also analyzed the association between the study groups and cardiovascular events, and found the group with higher indexed LAD and lower albumin was associated with an increase in cardiovascular events, which suggested LA enlargement and hypoalbuminemia might also have a synergic effect on the risk of adverse cardiovascular outcomes.

There were limitations to our study. LAD may not accurately reflect the true LA chamber in most patients. LA volume may be more precise, but LAD is a valid surrogate of LA size. LAD is more easily obtained than LA volume and measurement of LAD has already been the part of a routine echocardiographic evaluation. Some large population-based cohort studies, such as Strong Heart Study (1) and the Losartan Intervention For End point (13), used LAD as a surrogate of LA size. The majority of our patients were treated chronically with antihypertensive medications. For ethical reasons, we did not withdraw these medications. Hence, we could not exclude the influence of antihypertensive agents on our findings. In addition, it should be noted that serum albumin, a marker of visceral protein stores, can be influenced by several non-nutritional factors such as plasma volume expansion, albumin redistribution, exogenous loss, increased fractional catabolic rate, and decreased synthesis (36). Hence, the relationship between LAD and nutritional indices other than serum albumin, 
such as handgrip strength (37) and Subjective Global Assessment (38), needs further clarification. Finally, the quantitative assessment of proteinuria was not performed, so the impact of proteinuria could not be assessed properly in this study.

In conclusion, our study demonstrated that albumin was independently associated with indexed LAD and suggested that the combination of indexed LAD and serum albumin was independently associated with rapid renal progression and progression to dialysis. The association between indexed LAD and adverse renal outcomes might be introduced in part because of a direct association between albumin and indexed LAD. Assessments of serum albumin level and indexed LAD by echocardiography might be useful in identification of high risk group for adverse renal outcomes in patients with CKD.

\section{Acknowledgement}

The research presented in this article is supported by the grant from Kaohsiung Municipal Hsiao-Kang Hospital (kmhk-101-001), Kaohsiung Medical University, Kaohsiung, and the statistical work by the Department of Research Education and Training at Kaohsiung Municipal Hsiao-Kang Hospital.

\section{Competing Interests}

There is no competing interest in the information contained in the manuscript.

\section{References}

1. Kizer JR, Bella JN, Palmieri V, Liu JE, Best LG, Lee ET, Roman MJ, Devereux RB. Left atrial diameter as an independent predictor of first clinical cardiovascular events in middle-aged and elderly adults: the Strong Heart Study (SHS). Am Heart J 2006; 151: 412-8.

2. Stewart GA, Gansevoort RT, Mark PB, Rooney E, McDonagh TA, Dargie HJ, Stuart R, Rodger C, Jardine AG. Electrocardiographic abnormalities and uremic cardiomyopathy. Kidney Int 2005; 67: 217-26.

3. Chen SC, Chang JM, Liu WC, Huang JC, Tsai JC, Lin MY, Su HM, Hwang SJ, Chen HC. Echocardiographic parameters are independently associated with increased cardiovascular events in patients with chronic kidney disease. Nephrol Dial Transplant 2012; 27: 1064-70.

4. Kim SJ, Han SH, Park JT, Kim JK, Oh HJ, Yoo DE, Yoo TH, Kang SW, Choi KH. Left atrial volume is an independent predictor of mortality in CAPD patients. Nephrol Dial Transplant 2011; 26: 3732-9.

5. Furukawa M, Io H, Tanimoto M, Hagiwara S, Horikoshi S, Tomino Y. Predictive Factors Associated with the Period of Time before Initiation of Hemodialysis in CKD Stages 4 and 5. Nephron Clin Pract 2011; 117: c341-7.

6. Chen SC, Su HM, Hung CC, Chang JM, Liu WC, Tsai JC, Lin MY, Hwang SJ, Chen HC. Echocardiographic Parameters are Independently Associated with Rate of Renal Function Decline and Progression to Dialysis in Patients with Chronic Kidney Disease. Clin J Am Soc Nephrol 2011; 6: 2750-8.

7. Kim BS, Jeon DS, Shin MJ, Kim YO, Song HC, Lee SH, Kim SY, Choi EJ, Chang YS, Bang BK. Persistent elevation of C-reactive protein may predict cardiac hypertrophy and dysfunction in patients maintained on hemodialysis. Am J Nephrol 2005; 25: 189-95.

8. Vaziri SM, Larson MG, Lauer MS, Benjamin EJ, Levy D. Influence of blood pressure on left atrial size. The Framingham Heart Study. Hypertension 1995; 25: 1155-60.
9. Xu JZ, Wu SY, Yan YQ, Xie YS, Ren YR, Yin ZF, Shi CZ, Wang CQ. Left atrial diameter, flow-mediated dilation of brachial artery and target organ damage in Chinese patients with hypertension. J Hum Hypertens 2012; 26: 41-7.

10. Kursat S, Tekce H, Ekmekci C, Colak HB, Alici T. Relationship between the degree of malnutrition and echocardiographic parameters in hemodialysis patients. Nephron Clin Pract 2007; 106: c136-42.

11. Chen SC, Lin TH, Hsu PC, Chang JM, Lee CS, Tsai WC, Su HM, Voon WC, Chen HC. Impaired left ventricular systolic function and increased brachial-ankle pulse-wave velocity are independently associated with rapid renal function progression. Hypertens Res 2011; 34: 1052-8.

12. Silverstein DM. Inflammation in chronic kidney disease: role in the progression of renal and cardiovascular disease. Pediatr Nephrol 2009; 24: $1445-52$.

13. Gerdts E, Wachtell K, Omvik P, Otterstad JE, Oikarinen L, Boman K, Dahlöf B, Devereux RB. Left atrial size and risk of major cardiovascular events during antihypertensive treatment: losartan intervention for endpoint reduction in hypertension trial. Hypertension 2007; 49: 311-6.

14. Levey, AS, Coresh J, Bolton K, Culleton B, Harvey KS, Ikizler TA, Johnson CA, Kausz A, Kimmel PL, Kusek J, Levin A, Minaker KL, Nelson R, Rennke $\mathrm{H}$, Stettes $\mathrm{M}$, Witten B: Initiative KDOQ. K/DOQI clinical practice guidelines for chronic kidney disease: evaluation, classification, and stratification. Am J Kidney Dis 2002; 39: S1-266.

15. Devereux RB, Alonso DR, Lutas EM, Gottlieb GJ, Campo E, Sachs I, Reichek N. Echocardiographic assessment of left ventricular hypertrophy: comparison to necropsy findings. Am J Cardiol 1986; 57 : 450-8.

16. Mancia G, De Backer G, Dominiczak A, Cifkova R, Fagard R, Germano G, Grassi G, Heagerty AM, Kjeldsen SE, Laurent S, Narkiewicz K, Ruilope L, Rynkiewicz A, Schmieder RE, Boudier HA, Zanchetti A, Vahanian A, Camm J, De Caterina R, Dean V, Dickstein K, Filippatos G, Funck-Brentano C, Hellemans I, Kristensen SD, McGregor K, Sechtem U, Silber S, Tendera M, Widimsky P, Zamorano JL, Erdine S, Kiowski W, Agabiti-Rosei E, Ambrosioni E, Lindholm LH, Viigimaa M, Adamopoulos S, Agabiti-Rosei E, Ambrosioni E, Bertomeu V, Clement D, Erdine S, Farsang C, Gaita D, Lip G, Mallion JM, Manolis AJ, Nilsson PM, O'Brien E, Ponikowski P, Redon J, Ruschitzka F, Tamargo J, van Zwieten P, Waeber B, Williams B; Management of Arterial Hypertension of the European Society of Hypertension; European Society of Cardiology. 2007 Guidelines for the Management of Arterial Hypertension: The Task Force for the Management of Arterial Hypertension of the European Society of Hypertension (ESH) and of the European Society of Cardiology (ESC). J Hypertens 2007; 25: 1105-87.

17. Vickery S, Stevens PE, Dalton RN, van Lente F, Lamb EJ. Does the ID-MS traceable MDRD equation work and is it suitable for use with compensated Jaffe and enzymatic creatinine assays? Nephrol Dial Transplant 2006; 21: 2439-45.

18. Levey AS, Bosch JP, Lewis JB, Greene T, Rogers N, Roth D. A more accurate method to estimate glomerular filtration rate from serum creatinine: a new prediction equation. Modification of Diet in Renal Disease Study Group. Ann Intern Med 1999; 130: 461-70.

19. Shlipak MG, Katz R, Kestenbaum B, Siscovick D, Fried L, Newman A, Rifkin D, Sarnak MJ. Rapid decline of kidney function increases cardiovascular risk in the elderly. J Am Soc Nephrol 2009; 20: 2625-30.

20. Dolgos S, Hartmann A, Bollerslev J, Voros P, Rosivall L. The importance of body composition and dry weight assessments in patients with chronic kidney disease. Acta Physiol Hung 2011; 98: 105-16.

21. Wang AY, Sanderson J, Sea MM, Wang M, Lam CW, Li PK, Lui SF, Woo J. Important factors other than dialysis adequacy associated with inadequate dietary protein and energy intakes in patients receiving maintenance peritoneal dialysis. Am J Clin Nutr 2003; 77: 834-41.

22. Demirci MS, Demirci C, Ozdogan O, Kircelli F, Akcicek F, Basci A, Ok E, $\begin{array}{lll}\text { Ozkahya } & \text { M. Relations } & \text { between }\end{array}$ malnutrition-inflammation-atherosclerosis and volume status. The usefulness of bioimpedance analysis in peritoneal dialysis patients. Nephrol Dial Transplant 2011; 26: 1708-16.

23. Cheng LT, Tang W, Wang T. Strong association between volume status and nutritional status in peritoneal dialysis patients. Am J Kidney Dis 2005; 45: 891-902

24. Mitch WE, Goldberg AL. Mechanisms of muscle wasting. The role of the ubiquitin-proteasome pathway. N Engl J Med 1996; 335: 1897-905.

25. Anker SD, von Haehling S. Inflammatory mediators in chronic heart failure: an overview. Heart 2004; 90: 464-70.

26. Tousoulis D, Zisimos K, Antoniades C, Stefanadi E, Siasos G, Tsioufis C, Papageorgiou N, Vavouranakis E, Vlachopoulos C, Stefanadis C. Oxidative stress and inflammatory process in patients with atrial 
fibrillation: the role of left atrium distension. Int J Cardiol 2009; 136: 258-62.

27. Chung CM, Chu CM, Chang ST, Cheng HW, Yang TY, Wan PC, Pan KL, Lin YS, Hsu JT. Quantification of aortic stiffness to predict the degree of left ventricular diastolic function. Am J Med Sci 2010; 340: 468-73.

28. Gosse P, Pichot V, Guilhot M, Dauphinot V, Da Costa A, Barthelemy JC, Roche F. Relationship of cardiac involvement with arterial stiffness in a general population of 65-year-olds in the PROOF study. J Hypertens 2010; 28: 389-94.

29. Abhayaratna WP, Seward JB, Appleton CP, Douglas PS, Oh JK, Tajik AJ, Tsang TS. Left atrial size: physiologic determinants and clinical applications. J Am Coll Cardiol 2006; 47: 2357-63.

30. Douglas PS. The left atrium: a biomarker of chronic diastolic dysfunction and cardiovascular disease risk. J Am Coll Cardiol 2003; 42: 1206-7.

31. Tsang TS, Barnes ME, Gersh BJ, Bailey KR, Seward JB. Left atrial volume as a morphophysiologic expression of left ventricular diastolic dysfunction and relation to cardiovascular risk burden. Am J Cardiol 2002; 90: 1284-9.

32. Cuspidi C, Negri F, Sala C, Valerio C, Mancia G. Association of left atrial enlargement with left ventricular hypertrophy and diastolic dysfunction: a tissue Doppler study in echocardiographic practice. Blood Press 2012; 21: $24-30$.

33. Bock JS, Gottlieb SS. Cardiorenal syndrome: new perspectives. Circulation 2010; 121: 2592-600.

34. Ronco C. Cardiorenal syndromes: definition and classification. Contrib Nephrol 2010; 164: 33-8.

35. Johnson CA, Levey AS, Coresh J, Levin A, Lau J, Eknoyan G. Clinical practice guidelines for chronic kidney disease in adults: Part I. Definition, disease stages, evaluation, treatment, and risk factors. Am Fam Physician 2004; 70: 869-76.

36. Yeun JY, Kaysen GA. Factors influencing serum albumin in dialysis patients. Am J Kidney Dis 1998; 32: S118-25.

37. Waitzberg DL, Correia MI. Nutritional assessment in the hospitalized patient. Curr Opin Clin Nutr Metab Care 2003; 6: 531-8.

38. Young GA, Kopple JD, Lindholm B, Vonesh EF, De Vecchi A, Scalamogna A, Castelnova C, Oreopoulos DG, Anderson GH, Bergstrom J. Nutritional assessment of continuous ambulatory peritoneal dialysis patients: an international study. Am J Kidney Dis 1991; 17: 462-71. 